\title{
Research and practice of blended teaching of TCM English course fro $m$ the perspective of Educational Informatization
}

\section{Defang Zou}

Foreign Language Department, Guangxi University of Chinese Medicine (Nanning, Guangxi , 530200)

Supported by "Teaching Reform and Practice of Blended Teaching Model for TCM English Course under the Backgrou nd of Educational Informatization (NO. 2019A027)"

\begin{abstract}
In order to further promote the culture of Chinese medicine in our country, and to integrate with world medicine, in order to promote each other and develop together, the major of Chinese Medicine English came into being. However, under the traditional teaching mode, there are some problems in the teaching methods and methods of TCM English, which makes it difficult to improve the teaching effect. This article studies the mixed teaching mode of Chinese Medicine English in the information environment, and strives to make use of the complementary advantages of online teaching and traditional teaching, and improve the teaching quality of Chinese Medicine English with more effective teaching methods.

Keywords: Information environment; Chinese medicine English; blended teaching; research and practice

English for Chinese Medicine is a professional subject for better publicity and popularization of Chinese medicine knowledge, to promote Chinese medicine to the world, to let the world understand Chinese medicine, and to improve the traditional view of Chinese medicine. However, the teaching theories and practitioners of TCM English in our country are in the exploratory stage, which seriously restricts the development of TCM English teaching in terms of updating the content of teaching materials and the allocation of teachers. Combining many years of teaching experience, the author discusses and analyzes the mixed teaching of Chinese medicine English under the information environment from the actual teaching situation, expounds the problems in the traditional Chinese medicine English teaching, and proposes the mixed teaching reform ideas and related ideas based on this The measures are hoped to provide some useful suggestions for the majority of Chinese medical English teachers in actual teaching.
\end{abstract}

\section{Problems in Traditional Chinese Medicine English Teaching}

\subsection{It is difficult to learn and it is difficult to ensure effective pre-class preparation}

TCM English is not just a professional language learning. In addition to medical vocabulary, it also involves a large amount of TCM theoretical knowledge, which makes learning TCM English much more difficult. Therefore, it is difficult to ensure the effect of the preview if students do not have a professional teacher to follow up and guide them in the early stage of learning. If students encounter too many difficulties in the preview stage, they are prone to fear and weariness, which further reduces The effectiveness of the pre-class preparation stage is not conducive to the further study of students.

\subsection{The classroom teaching method is single and rigid, and the teaching effect is not ideal}

The teaching content of TCM English covers a wide range and has certain teaching requirements for students. In order to better complete the teaching tasks in a limited time, teachers often unconsciously become "full of words and words". In addition, in actual teaching, because of the large content and high density of the overall curriculum, it is 
difficult to communicate effectively with the students. In addition, the pre-class preview effect mentioned above is not ideal, which makes the teaching atmosphere of TCM English too boring. Naturally, efficiency cannot be effectively improved.

\subsection{Students are not interested in learning and cannot effectively complete the practice after class}

The content of TCM English is boring and boring. There is too much content to remember, and there are certain practical training requirements. This kind of heavy schoolwork pressure, if not well guided and motivated, can easily make students lose Interest to continue learning. However, many teachers do not pay attention to this problem whether in class or after class. All teaching design and teaching objectives are based on the teacher's teaching task, and the student's learning progress is not taken into consideration. The method of teaching the main body brings great learning pressure to students, which makes students not motivated to learn in class, practical training after class cannot be completed effectively, and the overall teaching effect of Chinese Medicine English cannot be effectively improved.

\section{Thoughts and measures for the reform of Chinese medicine English mixed teaching in the information environment}

\subsection{In an information environment, online teaching makes up for the shortcomings of traditional teaching}

As mentioned above, the effect of pre-class preparation in traditional teaching cannot be effectively guaranteed. This is because it is difficult to learn Chinese Medicine English. It is difficult to effectively carry out it without the guidance of professional teachers and relying solely on students. However, in an information environment, online teaching can effectively solve this problem. Teachers can use micro-classes to explain the preview content in advance, and students can use the Internet to learn before class, which not only solves the problem of students preparing for class It also greatly improves the effective time utilization rate of students, helps students use the fragmented time after class to consolidate what they have learned, and improves the learning efficiency of Chinese Medicine English.

\subsection{Under the information environment, blended teaching improves teaching efficiency}

Traditional teaching is limited by time and space, resulting in tight classroom teaching content, ignoring the subjectivity of students' teaching, and making teaching efficiency low. In the information environment, teachers can complement the advantages of online teaching and offline classroom teaching to improve the effectiveness of Chinese medicine English teaching. Teachers should use online teaching without time and space constraints, put a lot of basic teaching content on the Internet, guide students to learn and consolidate slowly in their free time after class, and at the same time establish a good online communication platform to collect And sort out the difficulties and problems encountered by students in online learning, integrate them and solve them in offline classroom teaching, and build classroom discussion links on this basis, promote effective communication between teachers and students, and activate the classroom teaching atmosphere. Improve classroom teaching effectiveness.

2.3 Under the information environment, blended teaching improves students' comprehensive ability

Traditional Chinese Medicine English is a subject that combines theory and practice. Students need to conduct practical training after understanding the basic theoretical knowledge. However, there are problems in traditional teaching that the practical effect is not obvious and teachers cannot effectively understand the students' learning situation. In the information environment, teachers can carry out a full range of practical training online, arrange various professional tests, quickly and effectively understand the basic learning level of each student, so as to re-improve and organize the teaching content. In addition, students will not become blind and goalless in the process of learning. Under the high-frequency online test, they can effectively understand their own shortcomings, consolidate and review in a targeted manner, quickly check deficiencies, and improve students comprehensively. Comprehensive ability of Chinese medicine English. 


\section{Concluding remarks}

In summary, there are many shortcomings in traditional Chinese medicine English teaching methods, which have greatly affected the development of Chinese medicine English. In the information environment, relevant education researchers have merged the advantages of online teaching and classroom teaching, changed the original teaching mode, and built a flexible learning environment for students, reducing the pressure of students' learning, and bringing them closer. To improve the relationship between teachers and students, and constantly optimize various teaching resources, improve students' enthusiasm for learning Chinese medicine English, strive to cultivate international Chinese medicine talents, and lay a good foundation for the international communication and exchange of Chinese medicine.

\section{References}

1. Wu Lianying, Feng Yuxiong. The design and case of multi-modal teaching mode in Chinese medicine English classroom[J]. Journal of Guangxi University of Traditional Chinese Medicine, 2012, 15(003): 111-113.

2. Zhao Dandan. Research on the introduction of traditional Chinese medicine culture in the English teaching of Chinese medicine colleges and universities in the Internet age[J]. Journal of Changchun University of Chinese Medicine, 2018, 034(005):964-966.

3. Wang Hongyang. Research on the reform of MOOC teaching in TCM English under the background of "Internet+" era[J]. Exam Weekly, 2017(79).12-13. 